\title{
Slow-plasmon resonant-nanostrip antennas: Analysis and demonstration
}

\section{Søndergaard, Thomas; Beermann, J.; Boltasseva, Alexandra; Bozhevolnyi, S.I.}

\section{Published in:}

Physical Review B Condensed Matter

Link to article, DOI:

10.1103/PhysRevB.77.115420

Publication date:

2008

Document Version

Publisher's PDF, also known as Version of record

Link back to DTU Orbit

Citation (APA):

Søndergaard, T., Beermann, J., Boltasseva, A., \& Bozhevolnyi, S. I. (2008). Slow-plasmon resonant-nanostrip antennas: Analysis and demonstration. Physical Review B Condensed Matter, 77(11), 115420.

https://doi.org/10.1103/PhysRevB.77.115420

\section{General rights}

Copyright and moral rights for the publications made accessible in the public portal are retained by the authors and/or other copyright owners and it is a condition of accessing publications that users recognise and abide by the legal requirements associated with these rights.

- Users may download and print one copy of any publication from the public portal for the purpose of private study or research.

- You may not further distribute the material or use it for any profit-making activity or commercial gain

- You may freely distribute the URL identifying the publication in the public portal 


\title{
Slow-plasmon resonant-nanostrip antennas: Analysis and demonstration
}

\author{
Thomas Søndergaard $, 1, *$ Jonas Beermann, ${ }^{1}$ Alexandra Boltasseva, ${ }^{2}$ and Sergey I. Bozhevolnyi ${ }^{1}$ \\ ${ }^{1}$ Department of Physics and Nanotechnology, Aalborg University, Skjernvej 4A, DK-9220 Aalborg Øst, Denmark \\ ${ }^{2}$ Research Center COM, Technical University of Denmark, Building 345v, DK-2800 Kgs. Lyngby, Denmark
}

(Received 13 November 2007; published 13 March 2008)

\begin{abstract}
Resonant scattering by gold nanostrip antennas due to constructive interference of counterpropagating slow surface plasmon polaritons (SPPs) is analyzed, including the quasistatic limit of ultrasmall antennas, and experimentally demonstrated. The phase of slow SPP reflection by strip ends is found from scattering spectra revealing the influence of non-SPP field components. Experimental scattering spectra of gold 17- and 23-nm-thin strips of 100-500 nm widths placed on quartz and covered with index-matching oil are in excellent agreement with theoretical predictions.
\end{abstract}

DOI: 10.1103/PhysRevB.77.115420

PACS number(s): 78.67.-n, 73.20.Mf, 42.25.-p, 71.36.+c

\section{INTRODUCTION}

Resonant interactions in metal nanostructures are widely exploited to strongly localize and enhance electromagnetic fields. Optical resonances of nanometer-thin metal wires of finite length (rods) have recently attracted considerable attention ${ }^{1,2,4,3,5}$ allowing one to efficiently control field enhancement effects via the structure geometry. Furthermore, nanorods can be thought of as optical analog to antennas in, e.g., the microwave regime ${ }^{6-10}$ with the difference that optical "half-wavelength" 9 or "quarter-wavelength"10 antennas can be considerably shorter than a half or quarter of the free-space wavelength due to the excitation of surface charge waves. ${ }^{9}$ Related quasi-two-dimensional resonator structures are metal nanostrips ${ }^{11}$ and gap-resonator structures. ${ }^{12}$ These resonant configurations have in common a very recent interpretation of the resonances as an effect of constructive interference between counterpropagating slow surface plasmon polaritons (SPPs) being bound to and propagating along either nanometer-thin metal wires ${ }^{9}$ and strips $^{11}$ or in nanometer-thin gaps between metal surfaces. ${ }^{12}$ This interpretation does account for the aforementioned decrease in the antenna and/or resonator length and relates the latter to the slow SPP wavelength. Similar to a microwave halfwavelength antenna, a few examples of the optical analog based on a thin rod matched a half of an effective SPP wavelength. ${ }^{9}$ This is not a general trend, however, as we found examples of the half-wavelength metal nanostrip resonator having the length of only one-third of the slow SPP wavelength. ${ }^{11}$ It is apparently necessary (and very important) in the resonator model to accurately take into account a phase shift acquired by the SPP upon reflection at rod, strip, or gap terminations.

Calculating the phase shift is not trivial and, while the reflection phase has been calculated in the case of ordinary and gap SPPs, ${ }^{13,14}$ no estimate of the phase exists for the reflection of slow SPPs at thin metal film terminations. In this work, the latter phase shift is determined, albeit indirectly, from scattering spectra calculated for gold nanostrips. The calculations are validated by showing excellent agreement with experimental spectra obtained with gold nanostrips in the course of carefully conducted measurements that also serve as an experimental demonstration of gold nanostrip antennas and/or resonators.

\section{ESTIMATE OF SLOW SURFACE PLASMON POLARITON REFLECTION PHASE}

It has been argued ${ }^{11}$ that resonance wavelengths $\lambda$ of metal strip resonators of thickness $t$ and width $w$ (see inset Fig. 1) are determined approximately by the following condition:

$$
w \frac{2 \pi}{\lambda} n_{\text {slow }}=m \pi-\phi
$$

where $n_{\text {slow }}$ is the real part of the mode index of slow SPPs bound to and propagating along a metal film with the same thickness $(t)$ as the strip, $m=1,2,3, \ldots$ is the order of the resonance, and $\phi$ is the phase of the reflection coefficient $(\bmod \pi)$ for (slow) SPP reflection at strip terminations. This model was verified without considering $\phi$ directly but by showing instead that increasing $w$ by $\lambda / 2 n_{\text {slow }}$ resulted in a strip exhibiting the resonance at a similar wavelength $\lambda .{ }^{11}$ However, the above condition cannot be used to predict the

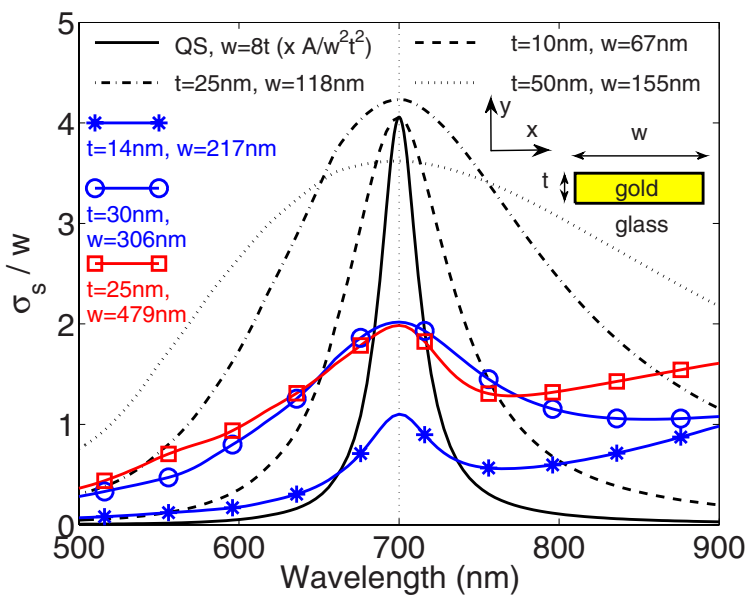

FIG. 1. (Color online) Scattering cross section vs wavelength for a plane $p$-polarized wave incident on a gold nano-strip of thickness $t$ and width $w$ embedded in glass. For $w=67-155$ and $479 \mathrm{~nm}$, and the quasistatic (QS) case, the incident wave propagates along the $y$ axis. For $w=217$ and $306 \mathrm{~nm}$, the incident wave propagates at $45^{\circ}$ relative to the $x$ axis. 
parameters $w$ and $t$ ensuring the resonance at a given $\lambda$, unless the phase $\phi$ is known. Here, we quantify the phase $\phi$ that satisfies the resonance condition [Eq. (1)] for various structure parameters.

Let us consider scattering spectra (Fig. 1) that were obtained using a Green function surface integral equation method for the magnetic field ${ }^{15}$ and a $p$-polarized plane electromagnetic wave of wavelength $\lambda$ (propagating in the $x y$ plane) being incident on a gold strip. The strip corners were rounded by $2 \mathrm{~nm}(0.2 t$ in the quasistatic case). The resonators with $w$ in the range $67-155 \mathrm{~nm}$ are first order resonances, those with $w=217$ and $306 \mathrm{~nm}$ are second order resonances, and the one with $w=479 \mathrm{~nm}$ is a third order resonance. We found that, in the limit of ultrathin structures $(t \leqslant 2 \mathrm{~nm})$, the scattering spectra except for a factor $w^{2} t^{2}$ depend only on the aspect ( $t$-to- $w$ ) ratio, becoming thereby similar to those of electrostatic (shape) resonances. The presented quasistatic case of $w=8 t$ is an $m=1$ resonance. From $t$ and $\lambda$, and the dielectric constants of the metal $\varepsilon_{m}$ (Ref. 16) and the surrounding dielectric $\varepsilon_{d}$ (here $\varepsilon_{d}=1.452^{2}$ ), we can calculate the mode index $n_{\text {slow }}$ either from the exact dispersion relation (see, e.g., Ref. 17) or simply by using an approximate formula for thin strips being an excellent approximation for $t \leqslant 200 \mathrm{~nm} .{ }^{18}$ In the quasistatic limit of very small $t$, we find ${ }^{18}$

$$
k_{\text {slow }}=\frac{2 \pi}{\lambda}\left(n_{\text {slow }}-i n_{\text {slow }}^{\prime \prime}\right) \approx-\frac{2 \varepsilon_{d}}{\varepsilon_{m} t},
$$

where the dependence on $\lambda$ enters only via the material dispersion (in $\varepsilon_{m}$ and $\varepsilon_{d}$ ). Consequently, the resonance condition [Eq. (1)] in the ultrathin limit [Eq. (2)] becomes indeed scale invariant, as is expected in the quasistatic limit. We also found that increasing $w(=8 t)$ by $2 \lambda / 2 n_{\text {slow }}$ estimated using Eq. (2) results again in a scattering resonance at approximately $\lambda=700 \mathrm{~nm}$. The factor of 2 is necessary as $m$ $=2,4, \ldots$ resonances do not radiate efficiently in this limit due to the field symmetry. The above considerations show that the concept of counterpropagating slow SPPs, i.e., retardation-based resonances, also applies to the quasistatic limit of ultrasmall resonators.

We can now extract $\phi$ by inserting $w, \lambda$ (at resonance), and $n_{\text {slow }}$ in Eq. (1). We have estimated $\phi$ for the peak scattering wavelengths 600,700 , and $800 \mathrm{~nm}$ versus $t$ (by adjusting $w$ ) for gold strips surrounded by air (Fig. 2) and glass (Fig. 3). Figures 2 and 3 were obtained using $n_{\text {slow }}$ obtained from the exact dispersion relation. ${ }^{17}$ Using the approximate formula $^{18}$ instead shifts the curves by no more than $0.025 \mathrm{rad}$.

Note that none of the first order resonators considered in Figs. 2 and 3 are close in length to a half of the slow SPP wavelength which requires $\phi=0(\pi)$. However, the phase decreases with increasing wavelength, which is not surprising as we know that $\phi \approx 0(\pi)$ for much longer wavelengths as, e.g., microwaves. The phase is also sensitive to the strip thickness, and by comparing Figs. 2 and 3, one notices the influence of the surrounding medium (glass or air) as well. Overall, we may conclude that the phase depends in a complicated way on $\lambda, t$, and $\varepsilon_{d}$.

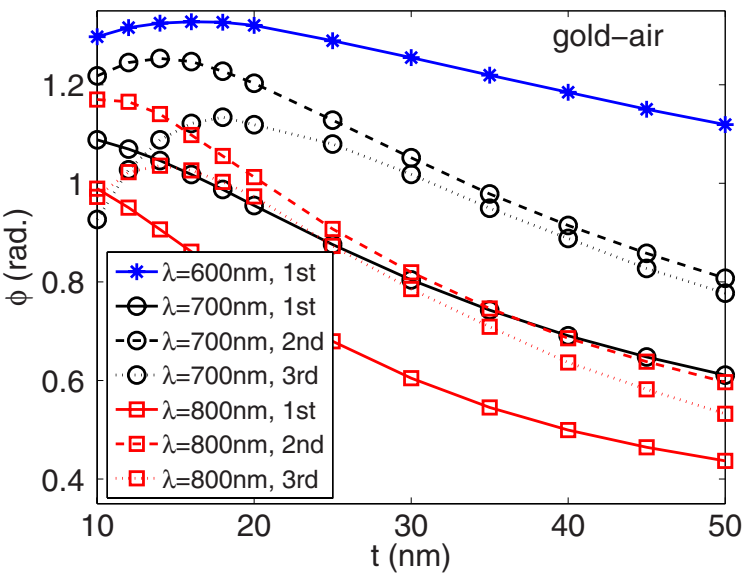

FIG. 2. (Color online) Reflection phase versus strip thickness $t$ according to Eq. (1) for a gold nanostrip surrounded by air for various resonance orders and the wavelengths 600,700 , and $800 \mathrm{~nm}$.

Surprisingly enough, at least from the first glance, the phases obtained using the first to third order resonances deviate from one another. This is related to the circumstance that not only the slow SPP fields are incident at strip terminations but also near field and other (non-SPP) propagating field components generated at one strip termination influence the total field at the other termination, which especially for small strip widths $w$ (first order resonators) influence the reflection of slow SPPs. At least for thicker strips $(\geqslant 20 \mathrm{~nm})$ the result for the first order appears shifted toward smaller values compared to second and third orders, where the results for the latter two orders agree reasonably well. This is consistent with the recent theory on interference resonances between slits, ${ }^{19,20}$ where the excitation of a surface plasmon polariton is also accompanied by a near field contribution at the site of excitation. We should mention that the angle of incidence can also affect the resulting phase of reflection because the incident field excites the (counterpropagating) SPPs at both strip ends. Turning this issue around, one can reason that, in general, the "true" reflection phase

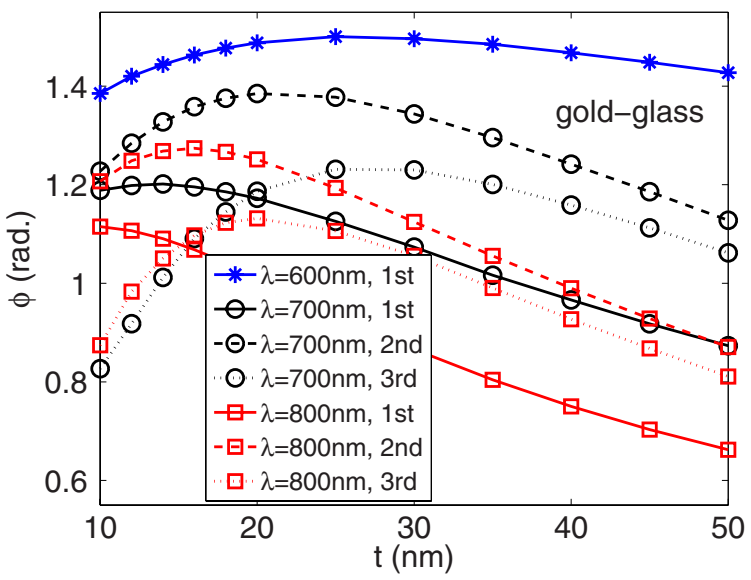

FIG. 3. (Color online) Same as in Fig. 2 but for a gold strip surrounded by glass. 


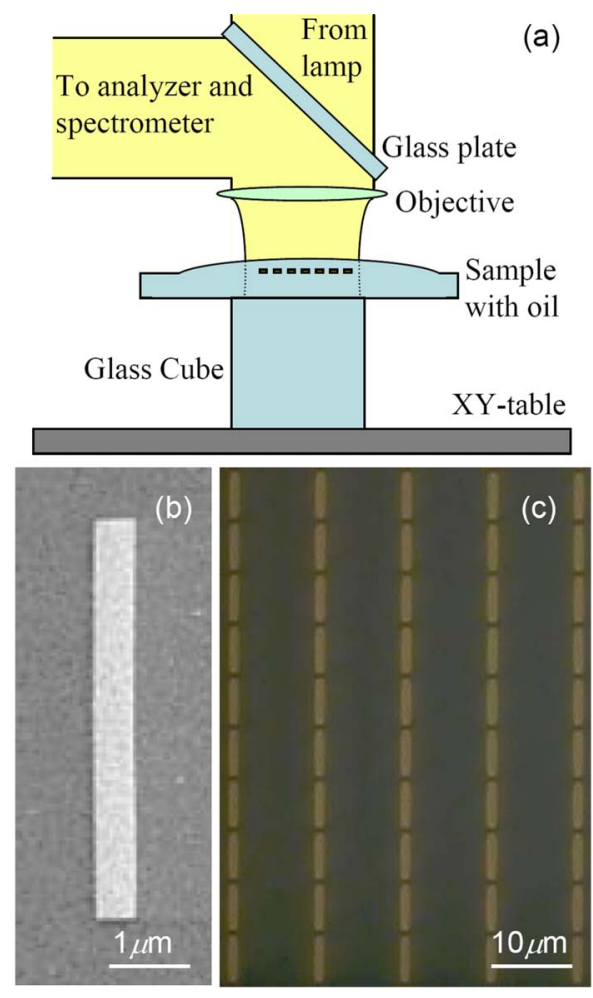

FIG. 4. (Color online) (a) Schematic of the experimental setup for reflection spectroscopy along with exemplary (b) SEM and (c) dark-field images from an area with 500-nm-wide and 23-nm-thick gold strip resonators on a quartz substrate.

can be determined only for semi-infinite strips not illuminated from the outside, so that the only incident wave (on the strip termination) is a slow SPP mode.

For the case of gold and glass (Fig. 3), wee see a phase deviation of approximately 0.25 between first and second order resonances for the wavelength $700 \mathrm{~nm}$ (just about the worst case), which should be compared to the range of $\pi$. The deviation is significant but not to an extent that renders Eq. (1) invalid as an approximate model of the physics of the resonances. The phase uncertainty of 0.25 transforms into an uncertainty [Eq. (1)] of less than $20 \mathrm{~nm}$ for the resonator width. However, for a resonator of small overall width, the wavelength uncertainty can be several times larger than the width uncertainty.

\section{EXPERIMENTS AND THEORY OF NANOSTRIP ANTENNA SCATTERING}

We have conducted careful investigations to experimentally demonstrate scattering resonances of strip resonators and validate the theoretical model. Several $100 \times 100 \mu \mathrm{m}^{2}$ arrays of gold strips, each containing 5- $\mu \mathrm{m}$-long strips of one fixed width $w$ and positioned at $1 \mu \mathrm{m}$ separation along and $10 \mu \mathrm{m}$ across the strips, were fabricated on quartz substrates by electron beam lithography and investigated using reflection spectroscopy. Our experimental setup for reflection spectroscopy [Fig. 4(a)] is based on a conventional optical microscope equipped with a Mitutoyo infinity-corrected long

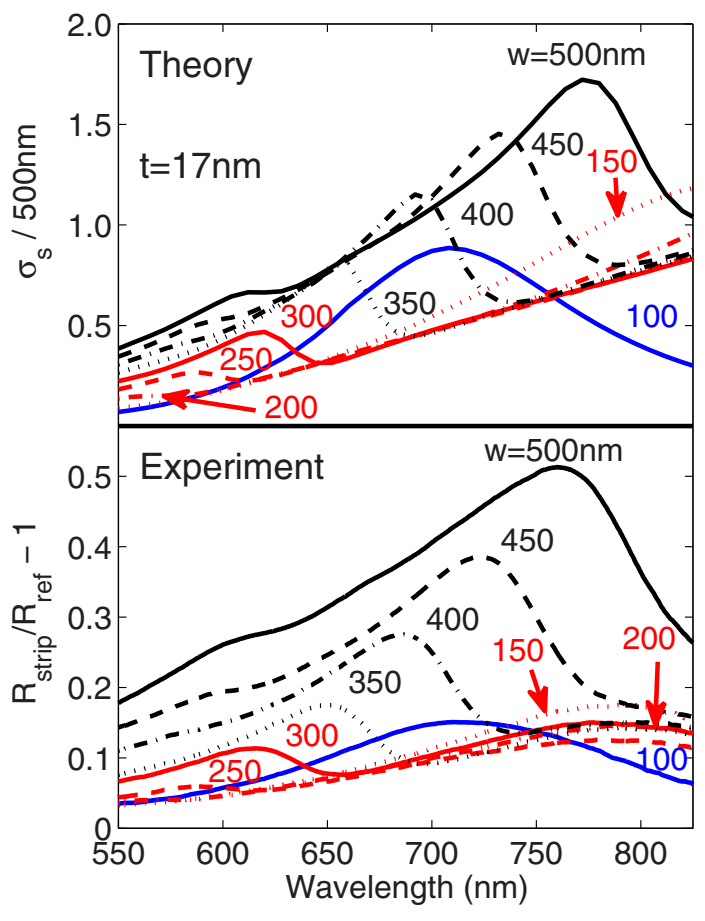

FIG. 5. (Color online) Measured and calculated scattering spectra for 17-nm-thin gold strips of different widths $w$ placed on quartz and covered by index-matching oil.

working distance objective $(50 \times$, numerical aperture 0.55$)$ and a possibility to access the light reflected from the sample with a fiber spectrometer recording between 540 and $1150 \mathrm{~nm}$. The illumination is a standard $50 \mathrm{~W}$ halogen bulb producing sufficient light up to $\sim 850 \mathrm{~nm}$, and a polarizer is inserted both before the sample and in front of the spectrometer entrance to fully control the polarization. The sample is covered with oil matching the refractive index (1.452) of the quartz substrate and positioned on top of a 1-cm-high glass cube, ensuring that only the reflected part of the light focused onto the gold strips and a small defocused part reflected from the top oil-air interface are collected by the spectrometer. For each spectrum measured from the strips we immediately record individual references at positions $\sim 250 \mu \mathrm{m}$ away from the concerned gold strip array in order to minimize the influence of any fluctuations in radiation spectra from the lamp. The gold strip arrays were fabricated for several strip widths from 100 to $500 \mathrm{~nm}$ at $50 \mathrm{~nm}$ increments, two different gold thicknesses (17 and $23 \mathrm{~nm}$ ), and each array being separated from the other by at least $500 \mu \mathrm{m}$. Both scanning electron microscopy and dark-field images from the sample show well defined and reproducible gold strip arrays [Figs. 4(b) and 4(c)]. Using the experimental data to compute the ratio $\left[\left(R_{\text {strip }}-R_{\text {ref }}\right) / R_{\text {ref }}\right]$, where $R_{\text {strip }}$ is the reflection measured from the strip array and $R_{r e f}$ is the corresponding reference, the experimental reflection spectra can be directly compared with theoretically estimated scattering cross sections for the same gold strip parameters (Figs. 5 and 6). It should be noted that the experimental spectra shown in Figs. 5 and 6 were obtained with $p$-polarized illumination (electric field across the strip) and that the spectra obtained with $s$-polarized illumination were essentially flat (as expected). 


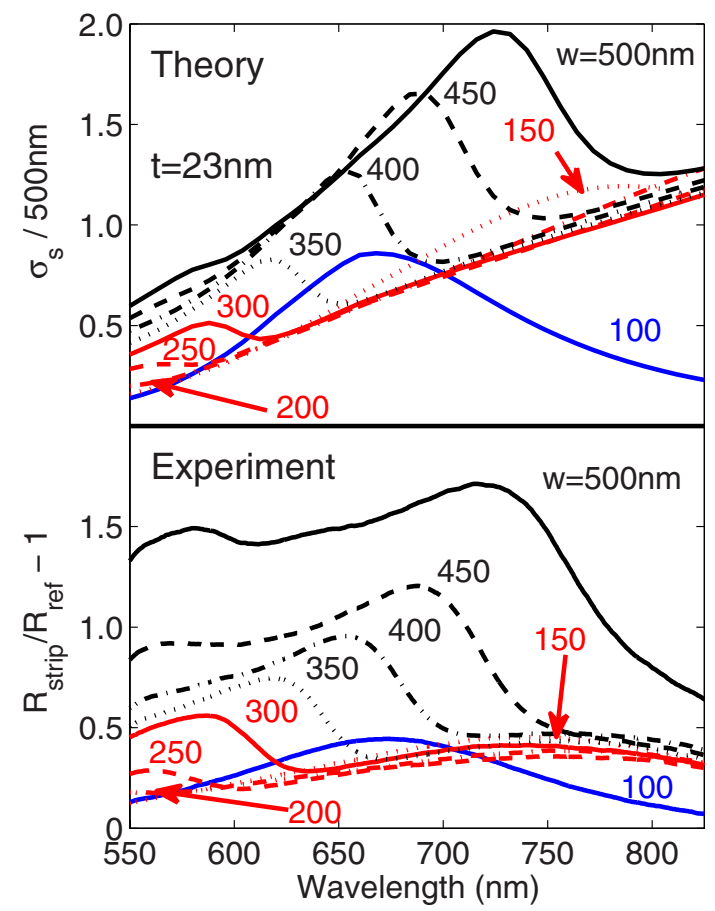

FIG. 6. (Color online) Same as Fig. 5 but for gold strips of thickness $23 \mathrm{~nm}$.

The nanostrips are placed in the focal plane of the illumination where the wave front is flat. The beam waist is furthermore much larger than a single strip (the whole array of strips is illuminated). Note that except for a scaling factor, the scattering calculations considering scattered light in all directions (Figs. 5 and 6) were similar to calculations including only scattered light in the limited angular range that the setup can collect.

We notice that for the same strip width, the wavelength of spectral peaks are clearly different for the two strip thicknesses 17 and $23 \mathrm{~nm}$. The reflection phase is not much different for these two thicknesses (Fig. 3) but $n_{\text {slow }}$ is smaller for the thicker strips, which results in smaller resonance wavelengths [Eq. (1)]. Also, in agreement with Eq. (1), the peaks shift to shorter wavelengths as the strip width decreases. The primary peak seen for widths in the range $200-500 \mathrm{~nm}$ is a third order resonance. The peaks seen for the widths 100 and $150 \mathrm{~nm}$ are first order resonances. No second order resonances are excited, neither in theory nor experiment, due to the symmetry mismatch of incident and resonant fields. They can be excited with the illumination at other angles of incidence. Also note that, e.g., for the waveguide width $500 \mathrm{~nm}$, a shoulder being a signature of a fifth order resonance is seen in both theory and experiment for wavelengths around $560 \mathrm{~nm}$ in Fig. 6 and wavelengths around $600 \mathrm{~nm}$ in Fig. 5. In the case of Fig. 6, the shoulder is apparently much more pronounced in the experiment than in the calculation. This is not the case in Fig. 5 though and the much more pronounced shoulder (Fig. 6) is probably a feature related to the specific sample.

Notice that the rather good agreement of theory and experiments is found even though the calculations neglect any variation of fields and structure along the $z$ axis, which is a very good approximation since $n_{\text {slow }}[\mathrm{Eq}$. (1)] is only marginally affected by a confinement over $5 \mu \mathrm{m}$ (the strip length) along the $z$ axis.

\section{CONCLUSION}

In conclusion, we have studied a model of nanometer-thin submicron-wide gold nanostrip resonators, where resonances are described as counterpropagating slow surface plasmon polaritons forming standing-wave resonances. We found that the picture of retardation-based resonances works even in the quasistatic limit of ultrasmall resonators. The model involves a reflection phase as slow SPPs are reflected at strip terminations. This phase was quantified via scattering calculations for strip resonators and was found to depend in a complicated way on the wavelength, the strip thickness, and the surrounding medium (glass or air). The estimated phase was found to depend on the resonance order. This nontrivial fact indicates apparently that slow SPP reflection at one strip termination is influenced by the presence of near field and nonSPP propagating field components both incident and originating from the other termination. Finally, gold nanostrip resonators were experimentally demonstrated via reflection spectroscopy of gold strips of thicknesses 17 and $23 \mathrm{~nm}$ surrounded by glass and index-matching oil, showing excellent agreement with the theoretical predictions. We believe that the considered physical phenomena are of general nature and thereby of crucial importance for other optical antenna and/or resonator configurations, such as thin metal wires and gap structures.

\section{ACKNOWLEDGMENTS}

The authors gratefully acknowledge support from the Danish Research Agency, the programme committee for nanoscience and technology, biotechnology, and information technology (NABIIT), Grant No. 2106-05-0033. T.S. acknowledges the Danish Research Council for Technology and Production, and S.I.B. the European Network of Excellence, PLASMO-NANO-DEVICES (FP6-2002-IST-1-507879). *ts@nano.aau.dk

${ }^{1}$ K. Imura, T. Nagahara, and H. Okamoto, J. Chem. Phys. 122, 154701 (2005).

${ }^{2}$ H. Ditlbacher, A. Hohenau, D. Wagner, U. Kreibig, M. Rogers, F. Hofer, F. R. Aussenegg, and J. R. Krenn, Phys. Rev. Lett. 95,
257403 (2005)

${ }^{3}$ F. Neubrech, T. Kolb, R. Lovrincic, G. Fahsold, A. Pucci, J. Aizpurua, T. W. Cornelius, M. E. Toimil-Molares, R. Neumann, and S. Karim, Appl. Phys. Lett. 89, 253104 (2006).

${ }^{4}$ T. Laroche and C. Girard, Appl. Phys. Lett. 89, 233119 (2006). 
${ }^{5}$ E. K. Payne, K. L. Shuford, S. Park, G. C. Schatz, and C. A. Mirkin, J. Phys. Chem. B 110, 2150 (2006).

${ }^{6}$ P. Mühlschlegel, H.-J. Eisler, O. J. F. Martin, B. Hecht, and D. W. Pohl, Science 308, 1607 (2005).

${ }^{7}$ J. Aizpurua, G. W. Bryant, L. J. Richter, F. J. Garcia de Abajo, B. K. Kelley, and T. Mallouk, Phys. Rev. B 71, 235420 (2005).

${ }^{8}$ E. Cubukcu, E. A. Kort, K. B. Crozier, and F. Capasso, Appl. Phys. Lett. 89, 093120 (2006).

${ }^{9}$ L. Novotny, Phys. Rev. Lett. 98, 266802 (2007).

${ }^{10}$ T. H. Taminiau, R. J. Moerland, F. B. Segerink, L. Kuipers, and N. F. van Hulst, Nano Lett. 7, 28 (2007).

${ }^{11}$ T. Søndergaard and S. I. Bozhevolnyi, Phys. Rev. B 75, 073402 (2007).
${ }^{12}$ H. T. Miyazaki and Y. Kurokawa, Phys. Rev. Lett. 96, 097401 (2006).

${ }^{13}$ R. Gordon, Phys. Rev. B 73, 153405 (2006).

${ }^{14}$ R. Gordon, Phys. Rev. B 74, 153417 (2006).

${ }^{15}$ T. Søndergaard, Phys. Status Solidi B 244, 3448 (2007).

${ }^{16}$ P. B. Johnson and R. W. Christy, Phys. Rev. B 6, 4370 (1972).

${ }^{17}$ E. N. Economou, Phys. Rev. 182, 539 (1969).

${ }^{18}$ S. I. Bozhevolnyi and T. Søndergaard, Opt. Express 15, 10869 (2007).

${ }^{19}$ G. Gay, O. Alloschery, B. Viaris De Lesegno, C. O’Dwyer, J. Weiner, and H. J. Lezec, Nat. Phys. 2, 262 (2006).

${ }^{20}$ G. Gay, O. Alloschery, J. Weiner, H. J. Lezec, C. O’Dwyer, M. Sukharev, and T. Seideman, Phys. Rev. E 75, 016612 (2007). 